\title{
Untersuchung von Strahlungsdefekten in GaP mittels Elektronenresonanz
}

\author{
H. Kimmel \\ Forschungslaboratorium der Siemens-Schuckertwerke AG, Erlangen \\ (Z. Naturforschg. 21 a, 526-528 [1966] ; eingegangen am 1. März 1966)
}

Herrn Prof. Dr. Trendelenburg zum 70. Geburtstag gewidmet

\begin{abstract}
Aus der Hyperfeinstruktur der Elektronenresonanz-Spektren von Elektronen- und Protonenbestrahltem GaP ergibt sich, daß Defekte auftreten, in denen ungepaarte Elektronen in Wechselwirkung entweder mit zwei Ga-Atomen oder mit zwei P-Atomen stehen. Bei den beobachteten Defekten dürfte es sich um die dem $\mathrm{Si}-\mathrm{A}$-Zentrum entsprechenden Defekte dieser im ZinkblendeGitter kristallisierenden binären Verbindung handeln.
\end{abstract}

Bei der Bestrahlung von Festkörpern mit energiereicher Strahlung entstehen verschiedene Defekte, denen ungepaarte Elektronen assoziiert sind und die deshalb paramagnetisches Verhalten zeigen. Es ist daher möglich, solche Defekte mit der Methode der Elektronenresonanz zu untersuchen und hierdurch Aussagen über deren strukturellen Aufbau zu erhalten. Das Resonanzspektrum solcher Zentren besteht in den weitaus meisten Fällen aus einer Vielfalt von Linien, die sich fast immer in ihrer Intensität, häufig in ihrer Halbwertsbreite und gelegentlich auch in ihrem Temperaturverhalten unterscheiden. Dabei sind im allgemeinen die Spektren von Defekten in Elementhalbleitern einfacher als diejenigen von Defekten in Verbindungshalbleitern. Das erklärt sich daraus, daß in Verbindungen mehr Arten von Fehlordnungen möglich sind als in Elementen. Beschränkt man sich beispielsweise auf die Fehlordnung einzelner Atome, also auf die einfachste Art von Strahlungsdefekten, dann gibt es bei Elementen nur die Möglichkeit, daß ein Atom statt auf einem Gitterplatz auf einem Zwischengitterplatz sitzt. In einer binären Verbindung $A B$ jedoch kann das A-Atom sowohl auf einem Zwischengitterplatz als auch auf einem B-Platz sitzen, desgleichen kann sich das B-Atom auf einem A-Platz oder auf einem Zwischengitterplatz befinden. Es gibt hier also bereits viermal so viele Arten einfachster Defekte wie bei Elementen. Daher ist es verständlich, daß am besten die Strahlungsdefekte in Silicium bekannt sind. Sie wurden im wesentlichen durch die grundlegenden Elektronenresonanz-Untersuchungen von Watkins u. a. ${ }^{1}$ und BeMski u. a. ${ }^{2}$ aufgeklärt.

1 G. D. Watkins, J. W. Corbett u. R. M. W Phys. 30, 1198 [1959]. - G. D. Watkins u. J. W. Corbett, Phys. Rev. 121, 1001 [1961].
Bei dem Versuch, Strahlungsdefekte in $\mathrm{A}^{\mathrm{III}} \mathrm{B}^{\mathrm{V}}$. Verbindungen mittels Elektronenresonanz zu untersuchen, stößt man im Vergleich zu Si auch noch aus anderen als den bereits genannten Gründen auf erhebliche Schwierigkeiten. Während Si aus zwei Isotopen besteht, von denen das mit etwa 95\% Häufigkeit auftretende den Kernspin 0 und das mit $5 \%$ Häufigkeit auftretende den Kernspin 1/2 hat, besitzen die Dreier- und Fünfer-Elemente im allgemeinen zwei Isotope mit Kernspins von $3 / 2$ oder höher. Das führt zu einem sehr großen Linienreichtum der Spektren.

Im Hinblick auf Isotopenzahl und Kernspin ist GaP für Elektronenresonanz-Untersuchungen am günstigsten: Ga besitzt zwei Isotope mit dem Kernspin $3 / 2$, aber mit unterschiedlichen Kernmomenten, und $\mathrm{P}$ besteht nur aus einem einzigen Isotop mit dem Kernspin 1/2. Die Untersuchungen wurden an polykristallinem Material durchgeführt. Die Proben waren entweder mit $3 \mathrm{MeV}$-Protonen oder mit $750 \mathrm{keV}$-Elektronen bei Zimmertemperatur bestrahlt. Die Elektronenresonanzspektren wurden bei $77^{\circ} \mathrm{K}$ mit einem Varian X-Band-Spektrometer bei $100 \mathrm{kHz}$ Modulation aufgenommen. Die Resonanzsignale waren nicht bei allen Proben gleich, was wohl auf eine unterschiedliche Reinheit der Materialien zurückzuführen sein dürfte. Bei mehreren Proben traten jedoch deutlich zwei Liniengruppen auf, wovon eine aus 3 Linien mit einem Intensitätsverhältnis von annähernd $1: 2: 1$ bestand und die andere aus 7 Linien, deren 3 innere Linien ein Intensitätsverhältnis von annähernd 3:4:3 aufwiesen. Die nach außen hin folgenden Linien der letztgenannten Gruppe waren

2 G. Bemski, G. Feher u. E. A. Gere, Bull. Am. Phys. Soc. 3, 135 [1958]. 
zunehmend verbreitert, was eine zusätzliche Verringerung ihrer Amplitude zur Folge hatte. Auf die Linienbreite der inneren Linien bezogen wäre für die Intensität der vorletzten Linien etwa die Hälfte von derjenigen der Zentrallinie anzusetzen, so daß sich demnach die Intensitäten der inneren 5 Linien der aus insgesamt 7 Linien bestehenden Gruppe wie 2:3:4:3:2 verhalten würden. Die äußersten beiden Linien der Gruppe waren sehr stark verbreitert und nur wenig über dem Rauschuntergrund, so daß eine auch nur einigermaßen zuverlässige Intensitätsrechnung nicht mehr möglich war.

Die erstgenannte, aus 3 Linien bestehende Gruppe mit einer den Binomialkoeffizienten entsprechenden Intensitätsverteilung deutet auf die symmetrische Wechselwirkung eines ungepaarten Elektrons mit zwei Kernen des Spins 1/2 hin. Es darf daher als sicher angenommen werden, daß es sich bei diesem Defekt um die symmetrische Beteiligung zweier PAtome handelt. Ebenso läßt sich bei der aus $7 \mathrm{Li}$ nien bestehenden Gruppe auf eine Wechselwirkung eines ungepaarten Elektrons mit zwei Kernen des Spins $3 / 2$ schließen, was eine Intensitätsfolge von $1: 2: 3: 4: 3: 2: 1$ ergibt. Es liegt hier also offensichtlich der analoge Defekt vor, jedoch unter Beteiligung zweier Ga-Atome. Ein möglicherweise auftretender ähnlicher Defekt, der aber aus zwei verschiedenen Atomen $\mathrm{Ga}$ und $\mathrm{P}$ aufgebaut ist, koninte nicht nachgewiesen werden.

Die beobachteten Defekte zeichnen sich demnach dadurch aus, daß sich die ungepaarten Elektronen in Elektronenbrücken aufhalten, welche sich nur zwischen zwei gleichen Atomen erstrecken. Im Gegensatz hierzu befinden sich im ungestörten Kristall, wo jedes Atom jeweils von 4 Atomen der anderen Art in Tetraedersymmetrie umgeben ist, die Elektronenbrücken stets zwischen ungleichen Atomen. Nun ist aber in Si ein Strahlungsdefekt bekannt, das $\mathrm{Si}-\mathrm{A}$ Zentrum ${ }^{1}$, bei dem sich eine Elektronenbrücke zwischen zwei Si-Atomen erstreckt, welche nicht auf unmittelbar benachbarten Gitterplätzen sitzen. Dieses Zentrum kommt dadurch zustande, daß ein Sauerstoffatom, welches ursprünglich als Verunreinigung auf einem Zwischengitterplatz war, eine durch Bestrahlung erzeugte und im Gitter diffundierende Leerstelle eingefangen hat. Von den vier dem Sauerstoffatom nächstbenachbarten Si-Atomen binden jetzt

3 G. W. Ludwig u. H. H. Woodbury, Solid State Phys. 13, 223 [1962]; Academic Press, New York. zwei zum Sauerstoff, während sich die anderen beiden gegenseitig binden. In der Brücke der letztgenannten Bindung befindet sich außer den beiden Bindungselektronen noch ein zusätzliches, ungepaartes Elektron, welches ein Resonanzsignal liefert. Ein Vergleich des $\mathrm{Si}-\mathrm{A}$-Zentrums mit dem GaP-Gitter zeigt, daß den sich gegenseitig bindenden Si-Atomen immer zwei Atome des gleichen Elementes bei GaP entsprechen. Es besteht daher Grund zu der Annahme, daß die beiden in $\mathrm{GaP}$ beobachteten Strahlungsdefekte diejenigen sind, welche in binären, im ZnS-Gitter kristallisierenden Verbindungen an Stelle des A-Zentrums auftreten. Es besteht jedoch kein Anhaltspunkt dafür, ob ebenso wie bei diesem auch in $\mathrm{GaP}$ der Sauerstoff eine wesentliche Rolle spielt, oder ob hier eine andere Verunreinigung an dessen Stelle tritt.

Ein Vergleich des $\mathrm{Si}-\mathrm{A}$-Zentrums mit dem GaPGitter zeigt ferner, daß es sich bei der Wechselwirkung des ungepaarten Elektrons mit zwei Ga-Atomen um einen Phosphordefekt handelt und bei einer Wechselwirkung mit zwei P-Atomen um einen Galliumdefekt. $\mathrm{Da}$ von den beiden Liniengruppen die 7er-Gruppe geringfügig an Intensität überwiegt, darf - sofern dem geringen Intensitätsüberschuß überhaupt eine Bedeutung zukommt - auf etwas mehr Phosphordefekte als Galliumdefekte geschlossen werden.

Nun ist der Abstand der einzelnen Hyperfeinstrukturlinien ein $\mathrm{Ma} \beta$ für die Aufenthaltswahrscheinlichkeit der entsprechenden ungepaarten Elektronen am Ort der jeweiligen Kerne ${ }^{3}$. Für die beiden Ga-Kerne (P-Defekt) ergab sich hieraus ein um den Faktor 1,5 größerer Wert als für die beiden P-Kerne (Ga-Defekt). Der Grund für dieses Verhalten dürfte der durch den ionogenen Bindungsanteil bedingte positive Ladungszustand der Ga-Atome bzw. die negative Ladung der P-Atome sein ${ }^{4}$. Da von den vier Bindungen der an einem Defekt von der Art des A-Zentrums beteiligten Gitteratome drei Bindungen praktisch unverändert geblieben sind, wird sich auch das Vorzeichen der effektiven Ladung nicht geändert haben.

Abschließend muß noch erwähnt werden, daß für eine genauere Analyse der beiden Strahlungsdefekte Einkristalle erforderlich wären. Insbesondere sollte an diesen eine vollständige Auflösung des im poly-

4 J. P. Suchet, J. Phys. Chem. Solids 21, 156 [1961]. - N. N. Sirota u. E. M. Gololobov, Soviet Phys.-Doklady 9, 477 [1964]. - H. Kimmes, Z. Naturforschg. 20 a, 359 [1965]. 
kristallinen Material als Gruppe von 7 Linien erscheinenden Spektrums der ungepaarten Elektronen zwischen den $\mathrm{Ga}$-Atompaaren möglich sein, welches entsprechend den beiden vorkommenden Ga-Isotopen aus 29 Einzellinien bestehen müßte.

Zur Beurteilung der Frage, durch welche Verunreinigungen die Bildung stabiler Defekte begünstigt wird, müßte wesentlich reineres Ausgangsmaterial zur Verfügung stehen. Auf Grund der bisherigen
Untersuchungen kann nur gesagt werden, daß auch bei $\mathrm{GaP}$ - ebenso wie bei $\mathrm{Si}$ - den Verunreinigungen eine entscheidende Bedeutung bei der Bildung von Strahlungsdefekten zukommt.

Für die Überlassung der bestrahlten Proben bin ich den Herren Bäuerlein und Dr. Wohlleben zu Dank verpflichtet. Herrn Schnitzke danke ich für die sorgfältige Durchführung der Messungen.

\title{
Magnetische, elektrische und thermische Eigenschaften von $\mathrm{FeGa}_{1,3}$
}

\author{
H. WAGINI \\ Forschungslaboratorium der Siemens-Schuckertwerke AG, Erlangen \\ (Z. Naturforschg. 21 a, 528-530 [1966] ; eingegangen am 2. Februar 1966) \\ Herrn Prof. Dr. Trendelenburg zum 70. Geburtstag gewidmet
}

\begin{abstract}
An der ferromagnetischen Verbindung $\mathrm{FeGa}_{1,3}$ wurde die Sättigungsmagnetisierung, der elektrische Widerstand, die absolute differentielle Thermospannung und die Wärmeleitfähigkeit oberhalb Zimmertemperatur gemessen. Es ergaben sich zwei Curie-Punkte bei $483^{\circ} \mathrm{K}$ und $697{ }^{\circ} \mathrm{K}$, die verschiedenen, gleichzeitig vorhandenen ferromagnetischen Strukturen zuzuordnen sind.
\end{abstract}

Unter den Schwermetallphasen, die mit III - VHalbleitern Eutektika bilden, findet man nach Untersuchungen von MüLler und WILHELM ${ }^{1}$ als neue ferromagnetische Verbindung $\mathrm{FeGa}_{1,3}$. Das $\mathrm{Zu}$ standsdiagramm des Systems $\mathrm{Fe}-\mathrm{Ga}$ wurde erst kürzlich von Dasarathy u. a. ${ }^{2}$ sowie Meissner u. a. ${ }^{3}$

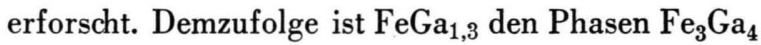
bzw. $\mathrm{Fe}_{8} \mathrm{Ga}_{11}$ gleichzusetzen. Die unterschiedlichen Stöchiometrien sind durch die Phasenbreite (ca. 56,6 bis 59,3 Atom-Proz. $\mathrm{Ga}^{2}$ ) bedingt. Die deutschen Autoren ${ }^{3}$ berichteten von einer monoklinen, C-flächenzentrierten Zelle, die Engländer ${ }^{2}$ konnten die Pulveraufnahmen tetragonal indizieren. Der Schmelzpunkt wurde mit ca. $906{ }^{\circ} \mathrm{C}$ und die Dichte mit $7,6 \mathrm{~g} / \mathrm{cm}^{3}$ angegeben. Weitere Daten sind bisher über $\mathrm{FeGa}_{1,3}$ nicht bekannt. Die vorliegende Arbeit berichtet über magnetische, elektrische und thermische Untersuchungen.

\section{Experimentelle Anordnung, Ergebnisse und Diskussion}

Obwohl die einkristallinen $\mathrm{FeGa}_{1,3}$-Einschlüsse aus dem eutektischen Gefüge isoliert werden kön-

1 A. Müller u. M. Wilhelm, J. Phys. Chem. Solids 26, 2029 [1965].

2 C. Dasarathy u. W. Hume-Rothery, Proc. Roy. Soc. London A 286, 141 [1965]. nen ${ }^{1}$, sind sie infolge ihrer geringen Abmessungen (maximale Länge $500 \mu$ ) den meisten physikalischen Messungen nicht zugänglich. Es wurde daher ein größeres Stück einer $\mathrm{Fe}-\mathrm{Ga}$-Legierung der Bruttozusammensetzung $\mathrm{FeGa}_{1,3}$ hergestellt. $\mathrm{Ga}(99,99 \%)$ und im $\mathrm{H}_{2}$-Strom geglühtes Carbonyleisen wurden im Atomverhältnis 1,3:1 zusammengeschmolzen und anschließend zum schnellen, ungerichteten Erstarren gebracht. Die metallographische Untersuchung des Schliffbildes zeigte mehrere Phasen. Nach mehrtägigem Tempern im Vakuum bei $800^{\circ}$ war das Material einphasig. Auch röntgenographisch waren die Proben den eutektischen Nadeleinschlüssen gleichwertig. Leider wies die Legierung kleine Poren auf, so daß die Meßergebnisse nur grobe Absolutwerte lieferten. Lediglich die geometrieunabhängige Thermospannung ist von diesen Fehlern nicht betroffen.

Für die magnetischen Untersuchungen wurde eine Probe $1,7 \mathrm{~mm} \times 1,7 \mathrm{~mm} \times 90 \mathrm{~mm}$ verwendet. Die Hysteresekurven wurden bei verschiedenen Temperaturen und $50 \mathrm{~Hz}$ mit der früher ${ }^{4}$ beschriebenen Apparatur oszillographisch aufgenommen. Auf diese Weise konnte nur die Anfangssuszeptibilität ermittelt

3 H. G. Meissner u. K. Schubert, Z. Metallk. 56, 523 [1965].

${ }^{4}$ H. W AGINI u. M. Wilhelm, Z. Naturforschg. 21 a, 329 [1966]. 